\title{
Hydrogen trapping in niobium-vanadium alloys
}

\author{
M A Pick†, S M Shapiro† and A M Stoneham $\ddagger$ \\ + Brookhaven National Laboratory, Upton, New York 11973, USA \\ $\ddagger$ AERE, Harwell, Didcot, Oxon OX110RA, UK
}

Received 22 July 1985 , in final form 21 October 1985

\begin{abstract}
We have measured the localised vibrational modes of hydrogen in a $\mathrm{Nb}_{0.93} \mathrm{~V}_{0.07}$ alloy. As one cools the sample for the first time to $10 \mathrm{~K}$ one observes that hydrogen is trapped at octahedral sites associated with two substitutional vanadium atoms. The trap energy is small $(10-20 \mathrm{meV})$ and less than the precipitation enthalpy. The phenomenon exhibits an interesting hysteresis. On repeated cooling cycles the hydrogen atoms tend to precipitate rather than stay in the trap sites. This is due to the formation of additional nucleation sites on the first cooling cycle.
\end{abstract}

\section{Introduction}

The presence of hydrogen trapped at lattice defects such as dislocations, interfaces, voids, interstitial and substitutional atoms, etc, can have important effects on materials. Embrittlement of structural materials and a pronounced influence on hydrogen recycling at the first wall of fusion reactors are but two of the many important examples. Detailed studies of hydrogen trapping have been performed, for example, on $\mathrm{Nb}$ and $\mathrm{Ta}$ metals with interstitial impurities such as oxygen and nitrogen (Baker and Birnbaum 1973, Pfeiffer and Wipf 1976, Richter and Springer 1978, Carstanjen 1980, Magerl et al 1983). Hydrogen trapping has been investigated by a variety of techniques including measurements of internal friction, hydrogen solubility, channeling, specific heat, diffusion constant and electrical resistivity. However, most of the techniques only provide indirect indications, if any, of the site location of the trapped hydrogen. Recently, it has been shown that the site location of hydrogen can be determined by an inelastic neutron scattering study of the local mode energies since these energies are characteristic of particular site geometries (Magerl et al 1983, Khoda-Bakhsh and Ross 1982). The inelastic spectra allow the detection of very low $\mathrm{H}$ concentrations - as low as $0.2 \%$ - which are beyond the range of detection by a normal diffraction study. In this paper we report results of an inelastic neutron scattering study of hydrogen trapping by substitutional $\mathrm{V}$ impurities in $\mathrm{Nb}$.

The reason we chose to study this particular system was because of its suitability in illustrating the sensitivity of the method to extract information about the trapping of hydrogen at substitutional sites as well as resolving the controversy created by the widely differing results reported on this particular system in the literature. Matsumoto et al (1975) report observing a decrease in the half-width of the $\mathrm{x}$-ray diffraction lines of a $\mathrm{Nb}-5 \% \mathrm{~V}$ alloy with the addition of hydrogen. They interpreted their results to mean that the interstitial hydrogen is strongly bound to the substitutional vanadium with a binding 
Table 1. A comparison of the trap energies of hydrogen in $\mathrm{NbV}$ alloys found by different authors and by various methods.

\begin{tabular}{lll}
\hline Method & Trap binding energy (eV) & References \\
\hline X-ray diffraction & 0.15 & Matsumoto et al (1975) \\
Solubility & 0.2 & Sasaki and Amano (1977) \\
Knight shift & 0.09 & Matsumoto (1977) \\
NMR & 0.08 & Mauger (1981) \\
Pressure against & $<0.07$ & Pick and Welch (1979) \\
$\quad$ composition & & \\
H vibrations & Notrapping & Magerl et al (1983) \\
Present analysis & $0.01-0.02$ (at pairs of V) & \\
\hline
\end{tabular}

energy $E_{\mathrm{B}}=0.15 \mathrm{eV}$. Actually the trapping of hydrogen atoms at point defects should not influence the half-width of the diffraction peaks, only their intensity, due to a rise in the static Debye-Waller factor. Sasaki and Amano (1977) attributed their observed increase in the hydrogen solubility of $\mathrm{Nb}$ with the addition of $\mathrm{V}$ to trapping with a binding energy of about $0.2 \mathrm{eV}$. An increase in the hydrogen solubility, however, does not necessarily imply trapping.

In an NMR study of the system $\mathrm{Nb}_{1-x} \mathrm{~V}_{x} \mathrm{H}_{x}$ (with $x=0.05$ ) Matsumoto (1977) measured the linewidth of the ${ }^{93} \mathrm{Nb}$ resonance absorption curve as well as the Knight shift of the ${ }^{51} \mathrm{~V}$ resonance. He also interpreted his results as due to the formation of vanadium-hydrogen pairs with a binding energy, estimated from the temperature dependence of the Knight shift at the vanadium site, of $0.09 \pm 0.05 \mathrm{eV}$. Mauger (1981), in a recent NMR study of the same system, deduced a binding energy of $0.08 \pm 0.01 \mathrm{eV}$.

Pick and Welch (1979), who studied the effect of vanadium additions to niobium on the equilibrium pressure-composition isotherms, estimated from the lack of a measurable effect that the binding energy of hydrogen to vanadium must be less than $0.07 \mathrm{eV}$.

Finally, Magerl et al (1983), who studied the hydrogen vibrations in a $\mathrm{NbV}_{0.008} \mathrm{H}_{0.005}$ alloy, report a shift in the phase boundary to lower temperatures due to the addition of the substitutional vanadium but found no clear indication of trapping at all.

A compilation of the various values including the result of the present analysis is shown in table 1 .

\section{Experiment}

Our present studies were on the alloy $\left(\mathrm{Nb}_{1-x} \mathrm{~V}_{x}\right) \mathrm{H}_{y}$ with $x=y=0.07$. The samples, obtained from the MRC Corporation, were cut into $5 \mathrm{~cm}$ lengths from a polycrystalline rod with a $1.2 \mathrm{~cm}$ nominal diameter. They were cleaned by electron beam heating to $2300 \mathrm{~K}$ in an ultrahigh vacuum $\left(10^{-10}\right.$ Torr) for approximately 24 hours. This method of preparing the samples is important in that virtually all gaseous impurities such as oxygen, nitrogen and carbon have been eliminated. Our samples are most likely cleaner than all those used in the earlier studies mentioned above. In order to introduce hydrogen into the samples without contamination, a thin layer of $\mathrm{Pd}$ was deposited on part of the sample in situ in the UHV chamber (Boes and Züchner 1976; see also Pick et al 1979). The sample was removed from the chamber and placed in a separate hydriding unit. There, a premeasured amount of hydrogen was readily absorbed by the metal at $\sim 80^{\circ} \mathrm{C}$ through the oxide-free Pd layer (Pfeiffer and Wipf 1978, Pick and Welch 1979). The Pd layer was subsequently removed by immersion in mercury. 
We measured both the inelastic spectra, in order to study the local modes and thereby the location of the hydrogen, as well as the elastic, or Bragg, spectra in order to be able to distinguish whether the hydrogen in the sample had precipitated in the $\beta$ phase or whether the sample remained in the $\alpha$ phase. If there were no hydrogen traps one would expect, on cooling the sample, to cross the $\alpha-(\alpha+\beta)$ phase boundary and to observe the appearance of the $\beta$-phase diffraction peaks in the elastic scattering spectra. If, however, one observed that on cooling the sample to very low temperatures the sample remained in the $\alpha$ phase, this would indicate that the hydrogen atoms were most likely trapped.

The inelastic neutron scattering experiments were performed at the High Flux Beam Reactor at Brookhaven National Laboratory. The well established technique was to use a cooled polycrystalline Be filter, which transmits neutrons with an energy less than $5.2 \mathrm{meV}$, as an analyser and to vary the incident neutron energy. The (002) reflection of beryllium served as a monochromator. A collimation of 40 minutes of arc was used between the reactor-monochromator, monochromator-sample and sample-detector. The resulting energy resolution is about $10 \mathrm{meV}$ for energy transfers $\Delta E=100 \mathrm{meV}$ and $15 \mathrm{meV}$ for $\Delta E=160 \mathrm{meV}$. The sample was mounted in an aluminium can filled with helium gas, which in turn was mounted on the cold finger of an Air Products Displex refrigerator. The temperature was varied from $10-300 \mathrm{~K}$.

The elastic diffraction spectra were obtained by simply removing the analysing filter and keeping the incident neutron energy fixed at $41.0 \mathrm{meV}$. We used a pyrolytic graphite filter to eliminate higher-order contamination. We usually only measured that portion of the diffraction pattern near the (200) Bragg peak of the $\alpha$ phase. The occurrence of $\beta$-phase precipitates is easily discernible by the appearance of an additional diffraction peak at slightly smaller $Q$ values. This reflects the somewhat expanded lattice of the $\beta$ phase. Other scans of the elastic diffraction spectra over a larger $Q$ range were performed with improved resolution and with similar results.

\section{Results}

The results are shown in figure 1 . The left side shows the inelastic scans and the right side the diffraction pattern in the neighbourhood of the (200) Bragg peak. The diffraction patterns were taken at the same temperature immediately after the inelastic scans.

Figure $1(a)$ shows the results obtained at $10 \mathrm{~K}$ after the initial cooling of the sample from room temperature over a period of about five hours. At this temperature one would expect the sample to be in the $(\alpha+\beta)$ two-phase region. One would then observe two diffraction peaks, the intensities of which would correspond to the amount of $\alpha$ and $\beta$ phase present. The conspicuous absence of the $\beta$-phase diffraction peak indicates that the hydrogen is trapped. The inelastic scan clearly shows four peaks and possibly more. This indicates that the hydrogen occupies at least two different sites. We associate the peaks at 113 and $164 \mathrm{meV}$ with the $\beta$ phase and those at 40 and $90 \mathrm{meV}$ with the hydrogen trapped by the substitutional vanadium.

The sample was then heated at $150 \mathrm{~K}$. At this temperature it is still within the $(\alpha+\beta)$ two-phase region. The results of the scans taken at that temperature are shown in figure $1(b)$. From the diffraction pattern, which now shows the $\beta$-phase diffraction peak, it is evident that the $\beta$ phase has now precipitated out (on heating the sample!). In the inelastic scan taken at this temperature the two lower energy peaks have been significantly 


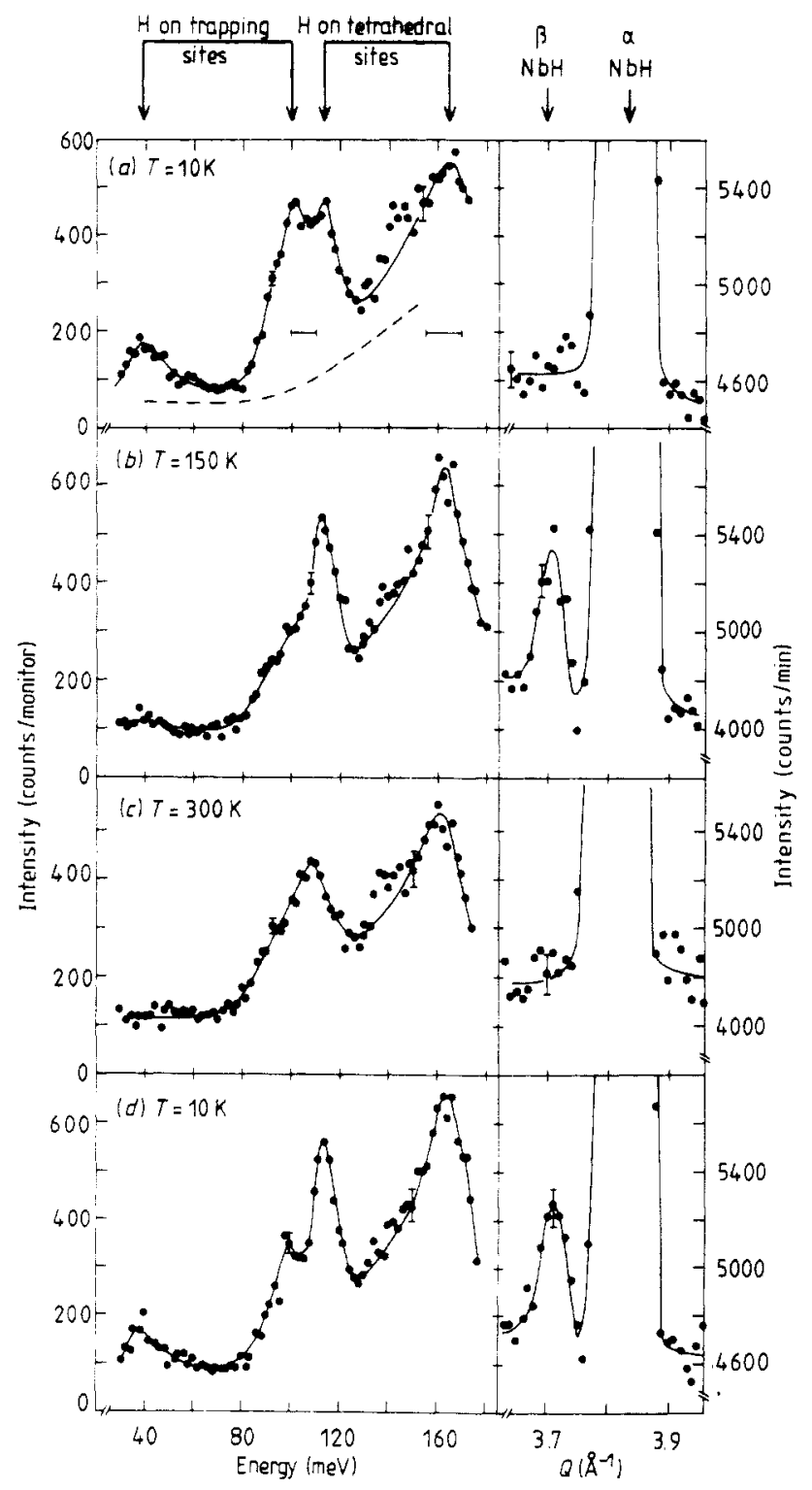

Figure 1. The left side of the figures shows the inelastic spectra due to hydrogen in the $\mathrm{Nb}_{0.93} \mathrm{~V}_{0.07}$ alloy, taken at different temperatures. At the top are indicated the approximate positions of the peaks for hydrogen sitting on tetrahedral interstitial sites as well as those found when hydrogen is trapped, apparently at octahedral interstitial sites, near a pair of vanadium atoms. On the right side are the elastic spectra, or Bragg peaks, in the vicinity of the (200) peak, taken at the same temperatures. On the top are indicated the approximate positions of the (200) Bragg peak of $\alpha-\mathrm{NbH}_{x}$ and that of the $\beta$ hydride when it is precipitated.

reduced in intensity. The other two peaks are dominant and increase slightly in intensity. The trapped hydrogen must therefore be associated with those peaks whose intensity has been reduced. 
At $300 \mathrm{~K}$ the sample is expected to be in the $\alpha$ phase. Indeed, the peak in the diffraction spectrum which characterises the $\beta$ phase has vanished. The two peaks in the inelastic spectra at 105 and $160 \mathrm{meV}$ are slightly shifted from those in the $\beta$ phase and are typical of the $\alpha$-phase spectrum of the pure $\mathrm{NbH}$ system. The widths of these peaks are also characteristically broader than those in the $\beta$ phase.

The spectra observed on recooling from 300 to $10 \mathrm{~K}$ (figure $1(d)$ ) differ from that of the initial cooling (figure $1(a)$ ). Both the inelastic and the diffraction spectra show more $\beta$ phase precipitate and less trapped hydrogen than after the initial cooling. We re-annealed the sample at high temperatures in the UHV system and reloaded with hydrogen as described above. After cooling to $10 \mathrm{~K}$, a spectrum very similar to figure $1(a)$ with significant trapping and no $\beta$ phase is observed.

\section{Discussion}

In order to understand the unusual trapping and hysteretic behaviour of the material we shall discuss the experimental findings from the point of view of a simple trapping mechanism and its consequences on the intensities of the peaks in the elastic and inelastic spectra. We first reiterate the following points:

(i) the 113 and $164 \mathrm{meV}$ peaks in the inelastic spectra correspond to the $\mathrm{NbH} \beta$-phase; i.e. they correspond to hydrogen atoms occupying tetrahedral interstitial sites ( $\mathrm{T}$ sites) which are either not associated with or not influenced by the presence of any vanadium;

(ii) the inelastic peaks at $\sim 40$ and $99 \mathrm{meV}$ are associated with hydrogen trapped in some way at vanadium sites. They probably correspond to hydrogen in octahedral sites with one or more vanadium neighbours (o sites). The energies are similar to those measured by Klauder et al (1979) on the concentrated alloy $\beta-\mathrm{VH}_{0.51}$ in which the hydrogen atoms are on octahedral sites.

The $\beta$-phase component can, therefore, be distinguished in two ways:

(i) by the relative intensity of the 113 and $164 \mathrm{meV}$ peaks and

(ii) by the characteristic diffraction peak.

From the intensities of the inelastic spectra of the sample after it was first cooled to $10 \mathrm{~K}$ one can deduce that about one half of the hydrogen atoms occupy the trapped sites. From the position and shape of the inelastic peaks one can tell that the other half are in the tetrahedral sites normally associated with the $\beta$ phase. We assume that the absence of the $\beta$-phase diffraction peak on first going to $10 \mathrm{~K}$ is because the regions of the $\beta$ phase are too small. It must therefore be the ripening of these small regions which gives rise to the $\beta$ diffraction peak on heating from 10 to $150 \mathrm{~K}$. However, the accompanying loss in intensity of the peaks at 40 and $99 \mathrm{meV}$, which we associate with the trapped sites, is probably due to a change in the equilibrium between the tetrahedral $(\mathrm{T}) \beta$-phase site and the $\mathrm{V}$-associated $O$ site. If this is so then since about one half of the trapped sites are depleted on heating to $150 \mathrm{~K}$, we expect that the binding energy of the $\mathrm{V}$-associated site, the configuration of which we will discuss below, will be of the order of $150 \mathrm{~K}(\sim 13 \mathrm{meV})$, i.e., a trap energy of only $0.01-0.02 \mathrm{eV}$ relative to the tetrahedral site in the $\alpha$ phase.

\subsection{Configuration of trapped hydrogen site}

The experimental evidence points towards hydrogen being trapped at an octahedral site associated in some way with one or more vanadium atoms. We shall discuss below the 
statistics of the possible configurations and suggest what we believe is the most likely configuration of this site.

In the $\mathrm{Nb}_{1-c} \mathrm{~V}_{c}$ alloy, which we assume to be random, there are both o sites and $\mathrm{T}$ sites. Each o site has six metal neighbours; each $\mathrm{T}$ site has four metal neighbours. For each host atom ( $\mathrm{Nb}$ or $\mathrm{V}$ ) there are three $\mathrm{O}$ sites and six $\mathrm{T}$ sites. We assume that the sites which trap hydrogen atoms are those octahedral sites with more than a 'critical' number of vanadium atom neighbours. This 'critical' number can be one or more. We shall assume that only two types of sites are occupied: O sites with more than the critical number of $V$ neighbours (we shall call the number of these sites $n_{0}$ ) and T sites with less than the critical number of $\mathrm{V}$ neighbours $\left(n_{\mathrm{t}}\right)$.

Supposing only one $V$ atom were sufficient to trap, i.e. the critical number were one,

$$
n_{0}=3 N\left[1-(1-c)^{6}\right]
$$

and

$$
n_{\mathrm{t}}=6 N(1-c)^{4}
$$

where $N$ is the total number of host atoms and the exponents 4 and 6 refer to the coordination numbers of the $O$ and $T$ sites, respectively. With our concentration of substitutional $\mathrm{V}$ atoms of $c=0.07 N$ this leads to

$$
n_{\mathrm{o}}=1.06 \mathrm{~N} \quad \text { and } \quad n_{\mathrm{t}}=4.69 \mathrm{~N} .
$$

From this result it is clear that in our case there are many more $n_{0}$ or trap sites than hydrogen atoms. Therefore there is no explanation of why not all of the hydrogen atoms are in the trapped sites or why the 40 and $90 \mathrm{meV}$ peaks are always so low in intensity.

The most likely option is that more than one $\mathrm{V}$ atom is required to produce a stable trap or $o$ site. The numbers of $o$ sites with various $V$ neighbours are given in table 2 . The numbers are to be compared with the number $0.07 \mathrm{~N}$ of hydrogen atoms. There are clearly too many sites with just one neighbour and too few with three neighbours.

If we assume that the trap site is an octahedral site with two vanadium neighbours then these two vanadium atoms can be distributed in some way on the two closest neighbouring sites and the four sites slightly further away. The fractions of these distributions are:

$\begin{array}{rll}2 / 30 & 0.011 N & \text { both close } \\ 16 / 30 & 0.088 N & \text { one close and one further } \\ 12 / 30 & 0.066 N & \text { both further. }\end{array}$

The site with the two closest neighbours occupied by vanadium atoms can be excluded as a trap site because there are too few of these sites. The most probable site is therefore one of the two remaining sites where, in the one case, one vanadium atom is close to the hydrogen atom and the other further away or the other case where both vanadium atoms are further away. It is one of these sites which weakly traps hydrogen (trap energy $\sim 0.01-0.02 \mathrm{eV}$ ) and which results in the localised hydrogen modes at $\sim 40$ and $99 \mathrm{meV}$.

This also explains the result of Magerl et al (1983), who found no appreciable trapping of hydrogen in their $\mathrm{NbV}_{0.008} \mathrm{H}_{0.005}$ alloy. In this case the fractions of the above trapping sites are 1.5 and $1.1 \times 10^{-3}$ respectively, which are both below the concentration of $5 \times 10^{-3}$ of hydrogen. In this case there are simply not sufficient trapping sites available. 
Table 2. The number of octahedral (o) sites with various numbers of vanadium neighbours, assuming a statistical distribution of vanadium in niobium. $N=$ number of host atoms, $c=$ concentration of $\mathrm{V}$ atoms $(c=0.07$ for the present sample).

\begin{tabular}{lll}
\hline $\begin{array}{l}\text { Number of } \\
\text { neighbours }\end{array}$ & $\begin{array}{l}\text { Number of } \\
\text { o sites }\end{array}$ & With $c=0.07$ \\
\hline 0 & $3 N(1-c)^{6}$ & $1.94 N$ \\
1 & $3 N 6 c(1-c)^{5}$ & $0.88 N$ \\
2 & $3 N 15 c^{2}(1-c)^{4}$ & $0.16 N$ \\
3 & $3 N 20 c^{3}(1-c)^{3}$ & $0.017 N$ \\
4 & $3 N 15 c^{4}(1-c)^{2}$ & $0.001 N$ \\
5 & $3 N 6 c^{5}(1-c)$ & $2.81 \times 10^{-5} N$ \\
6 & $3 N c^{6}$ & $1.18 \times 10^{-7} N$ \\
\hline
\end{tabular}

We shall now explain the unusual temperature hysteresis exhibited in figures $1(a)$ and (d). The sample has been thoroughly cleaned and annealed and therefore has a low density of nucleation sites (e.g. impurities, dislocations) for the formation of the $\beta$-phase hydride. As the sample is cooled down for the first time, the large distance between hydride nucleation sites prevents the system from reaching its thermodynamic equilibrium state in which virtually all the hydrogen is precipitated in the hydride phase. A large percentage of hydrogen atoms are trapped while diffusing towards a hydride nucleation site although the trap energy is well below the transformation enthalpy. Some small hydride regions do form, however, and these are distinguishable by their signature in the inelastic spectra. These regions are evidently too small to produce measurable diffraction peaks. On heating to $150 \mathrm{~K}$ these regions ripen and grow to form sizable precipitates and such regions are known to drastically increase the dislocation density by internal plastic deformation due to coherency stresses. If these defects are not annealed out, they provide new nucleation sites for the formation of $\beta$ hydride. Now the decreased mean distance between nucleation sites allows the system to approach its thermodynamic equilibrium state more closely on repeated cooling cycles. This is the reason for the observation that on the second and subsequent cooling cycles much less hydrogen is trapped and the $\beta$-phase precipitates are large and exhibit diffraction peaks. If the experiment were performed on a less pure or improperly cleaned sample, then trapping would not even be observed on the first cooling cycle because many nucleation sites would be present at the outset and this would favour the precipitation of the $\beta$ hydride.

The most probable reason for the high binding energy found by most other investigators is that their samples were contaminated with oxygen. The procedures used in the previous investigations (except those of Magerl et al 1983) to fabricate, homogenise and charge the alloys with hydrogen will tend to increase the oxygen concentration. This can be deduced from the known thermodynamic parameters for the solution of oxygen in niobium and vanadium (Fromm and Gebhardt 1976) as well as from experiments showing that effect (Makenas 1978). Furthermore, it is known that oxygen atoms in dilute substitutional niobium alloys tend to be strongly trapped by the substitutional solute atoms (Lauf and Altstetter 1979). In particular, oxygen is trapped by vanadium in niobium with a binding energy of approximately $0.55 \mathrm{eV}$ (Lauf and Altstetter 1979). Recent experimental EXAFS work by Pick (1985) indicates that such an agglomerate is a strong trap for hydrogen. 


\section{Acknowledgments}

Research at Bookhaven was performed under the auspices of the US Department of Energy, Division of Materials Sciences, Office of Basic Energy Sciences under Contract No DE-AC02-76CH0OO16.

\section{References}

Baker C and Birnbaum H K 1973 Acta Metall. 21865

Boes $N$ and Züchner H 1976 Z. Naturf. a 31754

Carstanjen H D 1980 Phys. Status Solidi a 5911

Fromm E and Gebhardt E 1976 Gase und Kohlenstoff in Metallen (Berlin: Springer)

Khoda-Bakhsh R and Ross D K 1982 J. Phys. F: Met. Phys. 1215

Klauder D, Lottner V and Scheuer H 1979 Solid State Commun. 32617

Lauf R J and Altstetter C J 1979 Acta Metall. 271157

Magerl A, Rush J J, Rowe J M, Richter D and Wipf H 1983 Phy's. Rev. B 27927

Makenas B J 1978 Metall. Trans. A 9738

Matsumoto T 1977 J. Phis Soc. Japan 421583

Matsumoto T, Sasaki Y and Hihara M 1975 J. Phys. Chem. 36215

Mauger P E 1981 PhD Thesis Cornell University

Pfeiffer G and Wipf H 1976 J. Phys. F: Met. Phys. 6167

Pick M A 1985 unpublished

Pick M A, Davenport J W, Strongin M and Dienes G J 1979 Phys. Rev, Lett. 43286

Pick M A and Welch D O 1979 Z. Phys. Chem. 11437

Richter D and Springer T 1978 Phys. Rev. B 18126

Sasaki Y and Amano M 1977 Hydrogen in Metals vol. 4 ed. P Axon (Oxford: Pergamon) 